\section{Generic iterative search-centre-shifting $K$-best sphere detection for rank-deficient SDM-OFDM systems}

\section{Wang, L. Xu, S. Chen and L. Hanzo}

A generic sphere-detection (SD) scheme is proposed, which substantially reduces the detection complexity by decomposing it into two stages, namely the generic iterative search-centre-update phase and the reduced-complexity search around it. This two-stage philosophy circumvents the high complexity of channel-coded soft-decision aided SDs.

Motivation: The sphere detector (SD) constitutes a computationally efficient solution to the maximum likelihood (ML) detection problem in uncoded multiple-input, multiple-output (MIMO) systems [1, 2]. However, when employed in an iterative detection aided channel coded system, the SD has to generate soft information for every transmitted bit, which requires the observation of a high number of hypotheses about the transmitted MIMO symbol, resulting in a potentially excessive complexity. To answer the question concerning how to maintain a near-ML performance with the aid of a small set of symbol hypotheses as posed in [1], we propose the centre-shifting scheme for SDs based on the novel discovery that, in contrast to the conventional SD [1, 2], the SD's search for the ML solution may be confined to a reduced search space centred at a point other than the MMSE solution.

Generic iterative centre-shifting SD: Conventionally, in the scenario of $(M \times N)$-element spatial-division-multiplexing (SDM) orthogonalfrequency-division-multiplexing (OFDM) MIMO systems using a constant modulus modulation constellation size of $M_{c}$, the ML solution can be found according to [2]:

$$
\hat{\boldsymbol{s}}_{M L}=\arg \min _{\breve{s} \in M_{c}^{M}}\left(\hat{\mathbf{s}}-\hat{x}_{c}\right)^{H}\left(\boldsymbol{H}^{H} \boldsymbol{H}+\sigma_{w}^{2} \boldsymbol{I}_{\mathcal{C}}\right)\left(\breve{\mathbf{s}}-\hat{\mathbf{x}}_{c}\right)
$$

by creating a reduced-size search space in the transmit MIMO-symbol domain centred on the MMSE solution $\hat{\boldsymbol{x}}_{c}=\left(\boldsymbol{H}^{H} \boldsymbol{H}+\sigma_{w}^{2} \boldsymbol{I}\right) \boldsymbol{H}^{H} \boldsymbol{y}$ by choosing an appropriate value for the maximum number of best MIMO-symbol candidates $K$ retained at each search level in the socalled $K$-best SD. Note that $\boldsymbol{H}$ is an $(N \times M)$-dimensional frequencydomain channel transfer factor (FDCHTF) matrix with each column representing the unique FDCHTF of the corresponding transmit antenna and $\sigma_{w}^{2}$ is the noise variance. This unique FDCHTF allows the separation of the $M$ SDM streams, even if using $N \leqslant M$ receiver antennas in the rank-deficient scenario considered.

During our investigations, we realised that it would be desirable to set the SD's search centre $\hat{x}_{c}$ to a high-probability MIMO signal constellation point, which may be expected to be closer to the true ML solution than the MMSE solution, because this would allow us to reduce the SD's search space by opting for a small value of $K$ and hence reduce its complexity. Our proposition is that the search centre be obtained by a more sophisticated detector, rather than by the conventional MMSE detector. This generic proposition turns the SD into a high-flexibility detector, which can be beneficially combined with arbitrarily sophisticated or low-complexity linear or nonlinear detectors. In other words, the total affordable computational complexity can be flexibly split between the SD's search-centre calculation phase and the search phase. We will demonstrate that an improved performance against complexity tradeoff emerges, if the search-centre calculation is iteratively updated, especially by the improved estimates of a channel decoder.

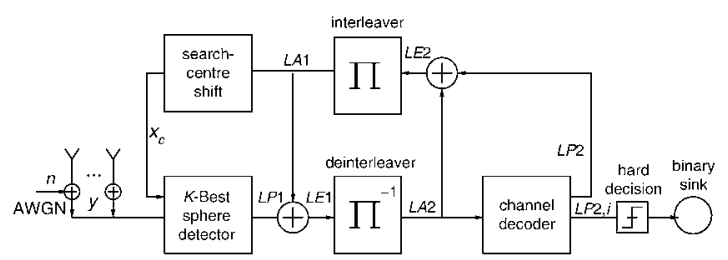

Fig. 1 Receiver architecture of generic iterative centre-shifting K-best SD scheme

Based on these observations, we surmise that the generic centre-shifting scheme applied for the SD is expected to become significantly more powerful, if it is employed in the scenario of the iterative detection aided channel coded system of Fig. 1. This is because the iterative process of obtaining a more accurate search centre is further aided by the channel decoder, which substantially improves the total error-correction capability of the iterative receiver. In comparison to the conventional SDaided iterative receiver of [1], the generic search-centre-shifting receiver proposed in Fig. 1 has an additional functional block for calculating the iteratively updated search centre $\hat{x}_{c}$. As an application example of the generic centre-shifting scheme, the MMSE soft-interference-cancellation (SIC-MMSE) algorithm of [3] is used. Hence, every time the search centre $x_{c}$ is updated, the SD is required to regenerate the candidate list [1], which is used to calculate the extrinsic LLRs delivered to the outer channel decoder of Fig. 1.

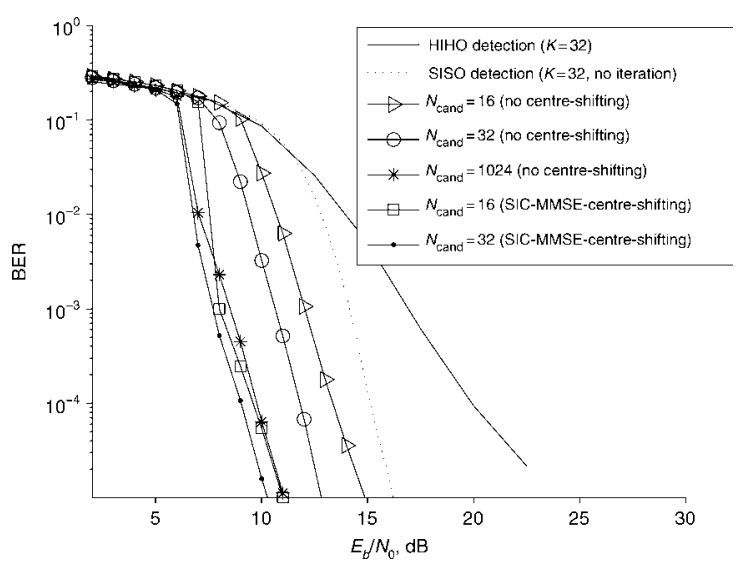

Fig. 2 BER performance improvement achieved by SIC-MMSE-aided iterative centre-shifting $K$-best $S D$ receiver in scenario of $(8 \times 4)$-element rankdeficient 4-QAM SDM/OFDM system

Application example: As shown in Fig. 2, the SIC-MMSE-aided iterative centre-shifting $K$-best SD using $K=16$ is capable of approaching the performance of the iterative SD dispensing with centre-shifting and hence using a large search space of $K=1024$, at a BER of $10^{-5}$ in the highly rank-deficient 4QAM scenario of $(8 \times 4)$ MIMO elements. Furthermore, for a fixed value of $K$, such as for example $K=32$ and for the same target BER of $10^{-5}$, we can observe that the iterative gain over the noniterative soft-input-soft-output (SISO) receiver was doubled to about $6 \mathrm{~dB}$ by the SIC-MMSE-aided centre-shifting scheme, when compared to that achieved by the iterative SD dispensing with centre-shifting.

Moreover, observe in Fig. 3 that the candidate list generation complexity of the SIC-MMSE centre-shifting-aided receiver, which is quantified in terms of the number of partial Euclidean distance (PED) evaluations, is well below that of the receiver using no centre-shifting for the SNR range spanning $2-12 \mathrm{~dB}$. This statement is valid, if our aim is to achieve the near-ML BER performance quantified in Fig. 2 by setting $K=N_{\text {cand }}=16$ in the presence of the centre-shifting scheme.

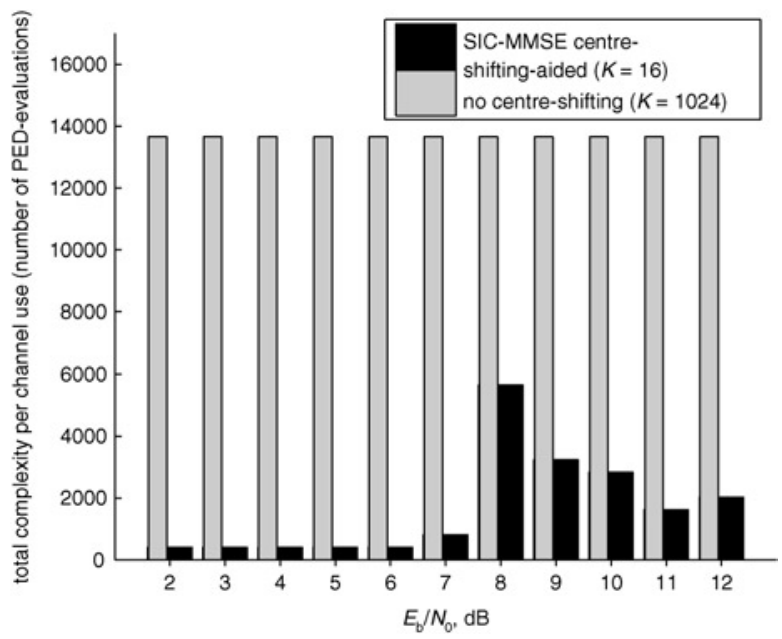

Fig. 3 Candidate-list-generation-related computational complexity histogram of SIC-MMSE-aided iterative centre-shifting K-best $S D(K=16)$ receiver in scenario of an $(8 \times 4)$-element rank-deficient 4-QAM SDM/ OFDM system 
Conclusions: The proposed near-ML SD finds the search-centre using an arbitrarily sophisticated or low-complexity detector and hence may reduce the detection-candidate-list-generation-related complexity in terms of the number of PED evaluations by an order of magnitude. As a further benefit, the computational complexity of the extrinsic LLR calculation quantified in terms of the number of objective function evaluations as shown in eqn. (12) of [1] was reduced by a factor of about 64 . The associated memory requirements were also reduced by a factor of 64 .

(C) The Institution of Engineering and Technology 2008

12 November 2007

Electronics Letters online no: 20083279

doi: 10.1049/el:20083279
L. Wang, L. Xu, S. Chen and L. Hanzo (School of ECS, University of Southampton, Southampton SO17 1BJ, United Kingdom)

E-mail: 1w05r@ecs.soton.ac.uk

\section{References}

1 Hochwald, B.M., and ten Brink, S.: 'Achieving near-capacity on a multiple-antenna channel', IEEE Trans. Commun., 2003, 51, pp. 389-399

2 Cui, T., and Tellambura, C.: 'An efficient generalized sphere decoder for rank-deficient MIMO systems', IEEE Commun. Lett., 2005, 9, pp. $423-425$

3 Tuchler, M., Singer, A.C., and Koetter, R.: 'Minimum mean squared error equalization using a priori information', IEEE Trans. Acoust. Speech Signal Process., 2002, 50, pp. 673-683 